\author{
${ }^{5}$ IPATIMUP-Institute of Molecular \\ Pathology and Immunology, University of Porto, \\ Porto, Portugal \\ E-mail: ESP@ous-hf.no
}

\section{References}

1 Carvalheira GM, Nozima BHN, Riggins GJ, et al. DDIT3, STT3A (ITM1), ARG2 and FAM129A (Niban, C1orf24) in diagnosing thyroid carcinoma: variables that may affect the performance of this antibody-based test and promise. Mod Pathol 2013;26:611-613.

2 Sigstad E, Paus E, Bjoro T, et al. The new molecular markers DDIT3, STT3A, ARG2 and FAM129A are not useful in diagnosing thyroid follicular tumors. Mod Pathol 2012;25:537-547.
3 Cerutti JM, Latini FR, Nakabashi C, et al. Diagnosis of suspicious thyroid nodules using four protein biomarkers. Clin Cancer Res 2006;12:3311-3318.

4 Rye PD, Nustad K, Stigbrand T. Tumor marker workshops. Tumour Biol 2003;24:165-171.

5 Willingham MC. Conditional epitopes. Is your antibody always specific? J Histochem Cytochem 1999;47: 1233-1236.

6 Adachi $\mathrm{H}$, Majima S, Kon S, et al. Niban gene is commonly expressed in the renal tumors: a new candidate marker for renal carcinogenesis. Oncogene 2004;23:3495-3500.

7 Haataja L, Gurlo T, Huang CJ, et al. Many commercially available antibodies for detection of CHOP expression as a marker of endoplasmic reticulum stress fail specificity evaluation. Cell Biochem Biophys 2008; 51:105-107.

\title{
Nested melanoma
}

Modern Pathology (2013) 26, 615-616; doi:10.1038/modpathol.2012.219

To the editor: The recent paper by Heinz Kutzner and co-authors introduces a new variant of superficial spreading melanoma composed predominantly of large hypercellular nests of melanocytes. ${ }^{1}$ The recognition of this particular pattern without a pagetoid element has been delayed as the histopathology masquerades as an unusual nevus. The clinical features, dermoscopy, the distinctive histopathology and now the application of comparative genomic hybridization, all help to identify this as a melanoma variant. Although the authors have linked this unusual nevoid melanoma to superficial spreading melanoma, this has necessitated a major change in the histopathological criteria traditionally applied to superficial spreading melanoma. There may be alternative distinctive pathways that need to be considered.

While exploring the relationship of atypical (dysplastic) nevi in the elderly to melanoma, ${ }^{2}$ the same experience emerged in the discrepancy between the clinical and dermoscopic findings that favoured a melanoma and the histopathological features which appeared nevoid. These melanocytic lesions when broad-based,often had criteria for defining transition to lentigo maligna ${ }^{3}$ or lentiginous melanoma. ${ }^{4}$ More recently, the results of FISH analysis have supported the existence of this controversial subtype of lentiginous melanoma ${ }^{5}$ that often evolves from atypical lentiginous nevi.

The atypical nevus of the elderly is an unstable nevus, and one variation which I have also observed $^{2}$ is the hypercellular nested variant described by the authors. I have reported these as in situ nevoid melanomas. The cellular morphology is usually associated with crowded small to medium hyperchromatic melanocytes. The progression of these atypical nested melanomas is often to a small cell (nevoid) melanoma, which may become desmoplastic.

Although from a clinical perspective the term superficial spreading melanoma is appropriate for this nested melanoma, at least some of these tumors may be linked to an aberrant nevus pathway seen in elderly individuals explaining their unusual pattern that resembles a bizarre nevus.

The authors have clearly defined and have highlighted a potentially important variant of melanoma that will now be recognized more frequently. The eventual classification of this melanoma needs further work in reference to the unstable atypical nevi seen in elderly individuals that may evolve as melanomas including this predominantly nested non lentiginous pathway.

\section{Disclosure/conflict of interest}

The authors declare no conflict of interest.

Steven Kossard
Department of Dermatopathology, Skin \& Cancer
Foundation Australia, Sydney, NSW, Australia
E-mail: skossard@scfa.edu.au

\section{References}

1 Kutzner H, Metzler G, Argenyi Z, et al. Histological and genetic evidence for a variant of superficial spreading melanoma composed predominantly of large nests. Mod Pathol 2012;25:838-845.

2 Kossard S. Atypical lentiginous junctional naevi of the elderly and melanoma. Australas J Dermatol 2002; 43:93-101. 
3 Kossard S, Commens C, Symons M, et al. Lentiginous dysplastic naevi in the elderly: a potential precursor for malignant melanoma. Australas J Dermatol 1991;32: $27-37$.

4 King R, Page RN, Googe PB, et al. Lentiginous melanoma: a histologic pattern of melanoma to be distinguished from lentiginous nevus. Mod Pathol 2005;18:1397-401.

5 Newman MD, Mirzabeigi M, Gerami P. Chromosomal copy number changes supporting the classification of lentiginous junctional melanoma of the elderly as a subtype of melanoma. Mod Pathol 2009;22:1258-1262. 